\section{Takayasu arteritis miss- diagnosed as thoracic outlet syndrome: A case report}

\section{Naseh Yousefi ${ }^{1}$, Peyman Roomizadeh ${ }^{1}$, Gholam Reza Raissi ${ }^{1}$, Shahram Sadeghi ${ }^{2}$, Katayoun Moradi ${ }^{1 *}$ and Shahram Rahimi-Dehgolan ${ }^{3 *}$}

${ }^{1}$ Neuromusculoskeletal Research Center, Department of Physical Medicine and Rehabilitation, Iran University of Medical Sciences (IUMS), Tehran, Iran

${ }^{2}$ Physical Medicine and Rehabilitation Research Center and Department, Shahid Beheshti University of Medical Sciences, Tehran, Iran

${ }^{3}$ Physical Medicine and Rehabilitation Department, Tehran University of Medical Sciences (TUMS), Tehran, Iran
Received: 27 August 2020

Accepted: 08 September, 2020

Published: 09 September, 2020

*Corresponding authors: Katayoun Moradi, MD, Department of Physical Medicine and Rehabilitation, Iran University of Medical Sciences, Tehran, Ira Postal Address: No. 1593747811, Firoozgar Hospital, Behafarin street, Valieasr square, Tehran, Iran Fax: +982188942970, Tel: +98[21]88908519

E-mail: moradi.ka@iums.ac.ir

Shahram Rahimi-Dehgolan, MD-MPH, Physical Medicine and Rehabilitation Department, Tehran University of Medical Sciences, Tehran, Iran

Postal Address: No. 1419733141, Physical Medicine and Rehabilitation Department, Keshavarz Blvd., Qarib street, Tehran, Iran. Tel/Fax: +982161190;

Email: shahram.rahimi.dehgolan@gmail.com

Keywords: Takayasu arteritis; Brachial plexopathy; Electrodiagnosis; Disputed neurogenic TOS

https://www.peertechz.com

Check for updates

\title{
Abstract
}

Takayasu Arteritis (TA) is a chronic idiopathic inflammatory vasculitis of unknown etiology that mainly involves the aorta and its major branches. In this study, we reported the case of a female patient with unilateral hand weakness that was initially misdiagnosed as TOS. However, at her most recent admission, the absence of radial pulse was noted which eventually led to the diagnosis of TA. Rarity of the disease, non-specific presentation and the absence of specific biochemical marker for TA made its early diagnosis difficult. We have tried to highlight the importance of conducting a thorough the physical examination when visiting patients with weakness, as well as the importance of considering rare differential diagnoses including TA in patients with unilateral limb paresis. TA might not manifest its typical constitutional symptoms preceding vessel involvement. Therefore a thorough clinical examination is pivotal in differentiating TA and TOS.

\section{Introduction}

Takayasu Arteritis (TA) is a chronic idiopathic inflammatory vasculitis of unknown etiology that mainly involves the aorta and its major branches [1]. TA more commonly affects females (gender ratio 10:1) under 40 years of age. It is a rare disease with worldwide annual incidence of 1-2 cases per million people [2] however, it has a remarkable racial preference as higher prevalence rates have been reported in Asian ethnic group $[3,4]$. The typical clinical presentation of TA is characterized by localized ischemic symptoms in the arms and hands related to inflammatory occlusion of large vessels of the upper limb. Arterial pulse might be absent in the affected arteries; therefore TA is also referred to as "pulseless disease" $[1,3]$. In some cases, the clinical presentation of TA might be non-specific and mimic a wide variety of possible differential diagnoses, making early detection difficult. In miss-diagnosed patients, symptoms gradually progress and may lead to serious complications. In this study, we report the case of a 46-yearold female patient with right upper limb pain and weakness, which was previously misdiagnosed as thoracic outlet syndrome (TOS).

\section{Case presentation}

A 46-year-old Persian woman, an intensive care unit nurse, was referred to the physical medicine and rehabilitation clinic of our hospital with right hand pain and weakness since one year ago. Her symptoms have gradually worsened over the last 6 months. She reported intermittent weakness and heaviness of right upper limb, exacerbated during physical activities such as using ambo-bag for her patients. She denied sensory symptoms such as paresthesia, hypoesthesia, numbness and tingling of the affected hands. There was no history of fever, arthralgia, rash, weight loss, vision loss, nausea, vomiting, 
dyspnea, or chest pain in recent months. There was no history of trauma to neck, shoulder or right upper limb. She denied heavy smoking, alcoholic habits and drug abuse. Her family history was negative. The patient had been attended to several specialists during this period and had been undergone numerous para-clinical evaluations including two Electrodiagnostic studies (EDx). The previous EDx had revealed normal nerve conduction and electromyography results at the first study and signs of mild carpal tunnel syndrome in the recent one. Neither of them could not explain her symptoms. Then she had been diagnosed with disputed-TOS initially and was prescribed with gabapentin, without significant improvement in her symptoms. No further evaluation for the etiology of weakness had been carried out.

On our physical examinations, the vital signs revealed a pulse rate of 66 beats/min and body temperature of 36.8 . The blood pressure was $100 / 70 \mathrm{mmHg}$ in the left arm and non-measurable in the right arm. The right hand was paler in color and cooler in palpation as compared to the left hand. Mild hypotrophy in right thenar and hypothenar areas were noted. No evidence of supraclavicular fullness was observed. Bruit over the right subclavian artery was noted. There was no necrotic lesion on fingers of either hand. The Adson's and Wright's tests were negative. The Roos test was positive as she was not able to complete the test due to fatigue and pain in her right forearm emerging within less than one minute of excursive. Manual muscle test (MMT) was normal in initial evaluation. Deep tendon reflexes were symmetric and normal in four extremities. Babinski's sign was not present bilaterally. A cranial nerve examination detected no abnormalities. The sensory examinations including light touch and pin prick test were normal. Other examinations were unremarkable.

The chest x-ray study was normal. A cervical Magnetic Resonance Imaging (MRI) revealed no significant abnormally. The laboratory analysis revealed hemoglobin level of $12 \mathrm{~g} / \mathrm{d}$, white-cell count of $15,500 / \mu \mathrm{L}$, platelet count of $222 \times 1000 / \mu \mathrm{l}$, Erythrocyte Sedimentation Rate (ESR) of $45 \mathrm{~mm} /$ hour and C-Reactive Protein (CRP) of $2+$. The blood glucose, serum creatinine, electrolytes, liver function tests, coagulation tests, and thyroid function tests were unremarkable. Serologic tests were negative for hepatitis panel, Antinuclear Antibody (ANA), Rheumatoid Factor (RF), Anti-Neutrophil Cytoplasmic Antibodies (ANCA), anti-phospholipid antibodies, anticardiolipin antibodies, and Human Immunodeficiency Virus (HIV) antibody.

A diagnosis of TA was suspected according to the absence of right radial pulse, a significant difference in blood pressures between the two arms, positive Roos test and subclavian bruits. A computed tomography angiography (CTA) of the carotid and subclavian arteries revealed considerably narrowed right subclavian artery as well as approximately $9^{\mathrm{cm}}$ elongation of artery occlusion after the origin of the right vertebral artery and a run off via axillary artery. On the basis of clinical features, physical examinations and CTA findings, the diagnosis of TA was made in accordance with American College of Rheumatology criteria [5]. A consultation with rheumatologist was performed; then oral prednisolone $50 \mathrm{mg} /$ day was started and her symptoms improved within a follow-up visit of two weeks.

\section{Discussion}

In this study, we reported the case of a female patient with unilateral hand weakness that was initially misdiagnosed as TOS. However, at her most recent admission, the absence of radial pulse was noted which eventually led to the diagnosis of TA. Rarity of the disease, non-specific presentation and the absence of specific biochemical marker for TA made its early diagnosis difficult. We have tried to highlight the importance of conducting a thorough physical examination when visiting patients with weakness, as well as the importance of considering rare differential diagnoses including TA in patients with unilateral limb paresis.

TA is an autoimmune disease that leads to inflammatory mononuclear cell infiltrates associated with giant cell formation in the lumen of large vessels, most commonly the aorta and subclavian arteries. The etiology of TA is not yet fully understood, but it has been suggested that overproduction of certain cytokines may play a role in the pathogenesis $[6,7]$. Like other large vessel vasculitis, TA is a systematic disease with both generalized and vascular symptoms. The generalized symptoms include fatigue, malaise, arthralgia, fever, weight loss and night sweats which may manifest prior to the onset of vessel involvement; however, these constitutional symptoms may not always be present $[8,9]$. To date, the diagnosis of TA is mainly based on the guideline laid by the American College of Rheumatology in 1990 [5]. This guideline requires having at least 3 of the 6 criteria as the following: age at the time of onset $\leq 40$ years, claudication in extremities, decreased brachial artery pulse, blood pressure difference $>10 \mathrm{~mm} \mathrm{Hg}$, bruit over subclavian arteries or aorta, and arteriogram abnormality. There is no unique biomarker for the diagnosis of TA at present. Our patient did not report the constitutional symptoms preceding limb paresis; however, she met the diagnostic criteria of TA as she had claudication in the right upper limb, absence of radial pulse, significant difference in blood pressure between the two arms, bruit over subclavian arteries, and narrowing of the right subclavian artery in CTA. These sign and symptoms have been neglected in her previous visits which led to delay in prompt diagnosis and treatment.

Clinical presentation of TA may mimic the presentation of TOS, as both diseases are characterized by unilateral limb pain and weakness, exacerbated during physical activity and ameliorated with rest. As an example, Dos Reis Neto et al. described a case of young woman with TOS that was initially miss-diagnosed as TA. They reported a 23-year-old female patient who presented with pain and intermittent claudication of the left forearm that was associated with ischemic lesions in her fingers, absence of radial pulse and non-measureable blood pressure of the affected arm. She also reported headache, malaise, fever and a weight loss of $5 \mathrm{Kg}$ during a short period. The index case had been previously miss-diagnosed as TA and was prescribed with oral prednisolone $60 \mathrm{mg} /$ day. Her symptoms did not improve with the corticosteroid treatment. On her latest admission, the chest radiograph revealed 
accessory cervical ribs and angiography of the upper limbs showed bilateral compression of the subclavian artery. The diagnosis of TOS was established and the patient underwent bilateral cervical rib excision which resulted in dramatic improvement in the symptoms [10]. It should be noted that the ischemic lesions of the fingers are not typically presented in TA as it primarily affects medium to large vessels $[1,3,10]$. On the other hand, the generalized and constitutional symptoms are not typically presented in TOS. Such cases in the literature, as well as our case, emphasize the overlap of symptoms between TA and TOS, thereby illustrating the importance of a detailed history and clinical examination to differentiate these entities accurately.

At the present time, corticosteroids are the mainstay of TA treatment. It has been proved to result in significant improvement especially for the systemic symptoms and an objective return of blood flow in the affected arteries [11]. Our patient responded well to a course of two weeks $50 \mathrm{mg} /$ day prednisolone treatment. In some occasions, patients might be resistant to corticosteroid treatments. In such cases, other immunosuppressive drugs including methotrexate, cyclophosphamide, azathioprine, cyclosporine, and mycophenolate mofetil could be recommended [12]. During recent years, some novel therapies including anti-tumor necrotizing factors (TNF) have also been suggested to be effective in patients with TA [13]. Surgical revascularization procedures are indicated in patients with severe hypertension due to renal artery obstruction, limb ischemia profoundly affecting daily activities, ischemia of cerebral vessels, congestive heart failure, coarctation of the aorta, cardiac ischemia due to stenosis in coronary arteries, dissected aneurysm, or in case of resistance to medical management $[14,15]$. TA should be considered as a rare but important differential diagnosis in any unilateral limb weakness. TA might not manifest its typical constitutional symptoms preceding vessel involvement. Therefore a thorough clinical examination is pivotal in differentiating TA and TOS

\section{Disclosure}

The authors have no proprietary interest in the materials presented herein. Our institutional (Iran University of Medical Sciences Ethics Committee) approval was required to publish the case details. In accordance with the ethics committee regulations, the patient was provided with all necessary information about the publication. Also, a written informed consent was obtained.

\section{References}

1. Seyahi E (2017) Takayasu arteritis: an update. Curr Opin Rheumatol 29: 51-56. Link: https://bit.ly/32dQTTU

2. Watts R, Al-Taiar A, Mooney J, Scott D, Macgregor A (2009) The epidemiology of Takayasu arteritis in the UK. Rheumatology 48: 1008-1011. Link: https://bit.ly/33dIVJp
3. Numano F, Kobayashi Y, Maruyama Y, Kakuta T, Miyata T, et al. (1996) Takayasu arteritis: clinical characteristics and the role of genetic factors in its pathogenesis. Vasc Med 1: 227-233. Link: https://bit.ly/2F2o4RB

4. Park YB, Hong SK, Choi KJ, Sohn DW, Oh BH, et al. (1992) Takayasu arteritis in Korea: clinical and angiographic features. Heart Vessels Suppl 7: 55-59. Link: https://bit.ly/3icFitz

5. Arend WP, Michel BA, Bloch DA, Hunder GG, Calabrese LH, et al. (1990) The American College of Rheumatology 1990 criteria for the classification of Takayasu arteritis. Arthritis Rheum 33: 1129-1134. Link: https://bit.ly/35kMSyO

6. Seko Y (2007) Giant cell and Takayasu arteritis. Curr Opin Rheumatol 19: 39 43. Link: https://bit.ly/33e1xZM

7. Wilke WS (1997) Large vessel vasculitis (giant cell arteritis, Takayasu arteritis) Baillieres Clin Rheumatol 11: 285-313. Link: https://bit.ly/3m0sN60

8. Numano $F$ (1997) Differences in clinical presentation and outcome in different countries for Takayasu's arteritis. Curr Opin Rheumatol 9: 12-15. Link: https://bit.ly/2ZjYOlw

9. Vanoli M, Bacchiani G, Origg L, Scorza R (2001) Takayasu's arteritis: a changing disease. J Nephrol 14: 497-505. Link: https://bit.ly/3hdrX2M

10. dos Reis Neto ET, Pucinelli ML, Silva de Souza AW, Sato El (2009) Thoracic outlet syndrome (TOS) mimicking Takayasu's arteritis--case report. Acta Reumatol Port 34: 96-101. Link: https://bit.ly/3bRdR6x

11. Koening CL, Langford CA (2006) Novel therapeutic strategies for large vessel vasculitis. Rheum Dis Clin North Am 32: 173-186. Link: https://bit.ly/3k2JTiq

12. Pérez-Esteban S, González-Gay MA, Castañeda S (2013) Therapeutic update in large vessel vasculitides. Rev Clin Esp (Barc) 213: 338-346. Link: https://bit.ly/33aixAr

13. Hoffman GS, Merkel PA, Brasington RD, Lenschow DJ, Liang P (2004) Antitumor necrosis factor therapy in patients with difficult to treat Takayasu arteritis. Arthritis Rheum 50: 2296-2304. Link: https://bit.ly/32cD5c1

14. Kerr GS, Hallahan CW, Giordano J, Leavitt RY, Fauci AS, et al. (1994) Takayasu arteritis. Ann Intern Med 120: 919-929. Link: https://bit.ly/2FbCheV

15. Liang P, Tan-Ong M, Hoffman GS (2004) Takayasu's arteritis: vascular interventions and outcomes. J Rheumatol 31: 102-106. Link: https://bit.ly/2ZjYjmE

Discover a bigger Impact and Visibility of your article publication with Peertechz Publications

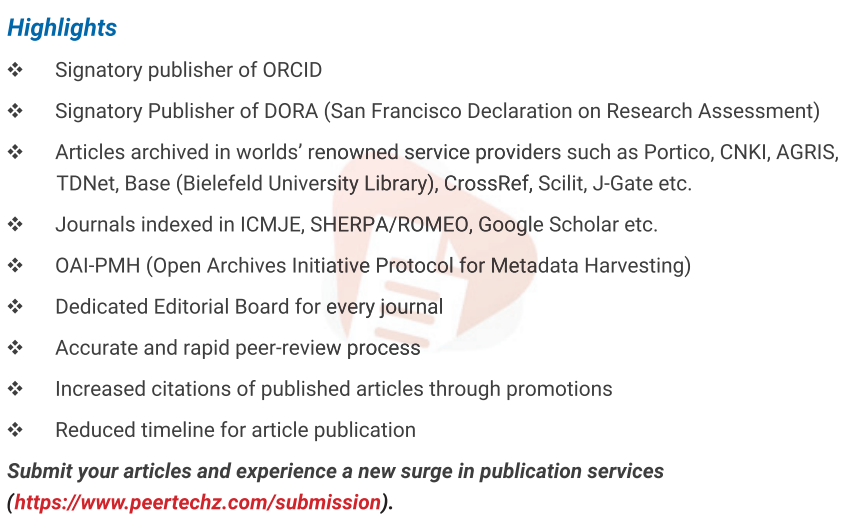

Peertechz journals wishes everlasting success in your every endeavours.

Copyright: @ 2020 Yousefi N, et al. This is an open-access article distributed under the terms of the Creative Commons Attribution License, which permits unrestricted use, distribution, and reproduction in any medium, provided the original author and source are credited.

Citation: Yousefi N, Roomizadeh P, Raissi GR, Sadeghi S, Dehgolan SR (2020) Takayasu arteritis miss-diagnosed as thoracic outlet syndrome: A case report. Ann Musculoskelet Med 4(2): 021-023. DOI: https://dx.doi.org/10.17352/amm.000023 